GHOsH A.

\title{
RICCI SOLITON AND RICCI ALMOST SOLITON WITHIN THE FRAMEWORK OF KENMOTSU MANIFOLD
}

\begin{abstract}
First, we prove that if the Reeb vector field $\xi$ of a Kenmotsu manifold $M$ leaves the Ricci operator $Q$ invariant, then $M$ is Einstein. Next, we study Kenmotsu manifold whose metric represents a Ricci soliton and prove that it is expanding. Moreover, the soliton is trivial (Einstein) if either (i) $V$ is a contact vector field, or (ii) the Reeb vector field $\xi$ leaves the scalar curvature invariant. Finally, it is shown that if the metric of a Kenmotsu manifold represents a gradient Ricci almost soliton, then it is $\eta$-Einstein and the soliton is expanding. We also exhibited some examples of Kenmotsu manifold that admit Ricci almost solitons.
\end{abstract}

Key words and phrases: Kenmotsu manifold, Ricci almost soliton, warped product.

Department of Mathematics, Chandernagore College, 712 136, Hooghly, India

E-mail: aghosh_70@yahoo.com

\section{INTRODUCTION}

In the recent years, there has been a growing interest in the study of Riemannian manifolds endowed with a metric which satisfies some structural equations involving Ricci curvature and some globally defined vector fields. Sometimes these are also appear as a solution of some geometric flows [5] and [7]. For instance, a Ricci soliton appears as a special (self-similar) solution of the Hamilton's Ricci flow [11]:

$$
\frac{\partial}{\partial t} g_{i j}=-2 S_{i j}
$$

A Ricci soliton is a smooth manifold $M$ together with a Riemannian metric $g$ that satisfies

$$
\left(£_{V} g\right)(X, Y)+2 S(X, Y)=2 \lambda g(X, Y)
$$

where $V$ is a vector field known as the potential vector field, $£_{V}$ denotes the Lie-derivative operator along a vector field $V, S$ is the Ricci tensor, $\lambda$ is a constant, and $X, Y$ arbitrary vector fields on $M$. This is also considered as a generalized fixed point of the Hamilton's Ricci flow, viewed as a dynamical system, on the space of Riemannian metrics modulo diffeomorphisms and scalings.

$\mathrm{y} \Delta \mathrm{K} 514.764 .226,514.154$

2010 Mathematics Subject Classification: 53C25, 53C15, 53D15.

This work has been supported by the UGC(India) under the scheme Minor Research Project in Science, Sanction No. PSW-018/15-16, Dated 15-11-2016. 
Recently, in [16] Pigola et al. extended the notion of Ricci soliton on a Riemannian manifold by allowing the constant $\lambda$ to be smooth function in the defining condition of the Ricci soliton (1). It is said to be shrinking, steady, or expanding according as $\lambda$ is positive, zero, or negative respectively. If the potential vector field $V$ is the gradient of a potential function $f$, then $g$ is called a gradient Ricci almost soliton. In this case, the soliton equation (1) transforms into

$$
\nabla_{X} D f+Q X=\lambda X
$$

where $D$ is the gradient operator with respect to the metric $g, Q$ is the Ricci operator asoociated with the Ricci tensor $S$, i.e., $S(X, Y)=g(Q X, Y), X, Y$ are arbitrary vector fields on $M$. Both equations (1) and (2) can be considered as a generalization of the Einstein equation $S=\lambda g$ and reduce to this latter in case $V$ or $D f$ are Killing vector fields. When $V=0$ or $\mathrm{f}$ is constant we say the underlying Einstein manifold a trivial Ricci soliton. On a compact Riemannian manifold a Ricci soliton is always a gradient Ricci soliton [15]. Ricci solitons are of interest to physists as well and are known as quasi Einstein metrics in the physics literature [8]. Some aspects of compact Ricci almost soliton may be found in $[3,4,17]$. In particular, in [4] it is proved that any compact Ricci almost soliton is gradient provided its scalar curvature is constant (see also [17]).

In [13], a new class of almost contact metric manifolds was introduced and studied, which is known as Kenmotsu manifold. This type of manifold is very closely related to the warped product spaces. Actually, the warped product space $\mathbb{R} \times{ }_{\sigma} N^{2 n}$ with the warping function $\sigma(t)=c e^{t}$ on the real line $\mathbb{R}$, and $N^{2 n}$ is Kähler admits such structure. Conversely, every point of a Kenmotsu manifold has a neighbourhood which is locally a warped product $(-\varepsilon, \varepsilon) \times_{\sigma} N^{2 n}$ where $\sigma(t)=c e^{t}$ is a function on the open interval. It is interesting to notice that a Kenmotsu manifold can not be compact because it satisfies div $\xi=2 n$. Recently, the author [9] studied Kenmotsu 3-metric as a Ricci soliton and proved that it is of constant negative curvature -1 . The existence of such metric has also been confirmed on the warped product of a Riemann surface $\mathrm{N}$ of constant negative curvature (a Kähler manifold) with the real line. For higher dimensions it is proved that "If the metric of an $\eta$-Einstein Kenmotsu manifold is a Ricci soliton, then it is necessarily an Einstein manifold" (see [10]). These results intrigues us to consider Kenmotsu metric as a Ricci soliton. The organization of the paper is as follows. After recalling some basic definitions and formulas in Section 2, we study Kenmotsu manifold satisfying $£_{\xi} Q=0$ in Section 3. In Section 4, we consider Kenmotsu metric as Ricci soliton. Finally, we study Kenmotsu metric as Ricci almost solitons.

\section{Preliminaries on KenMotsu Manifold}

In this section, we recall the definitions and fundamental formulas on Kenmotsu manifolds. Let $M$ be a smooth manifold of dimension $(2 n+1)$. Then $M$ is said to be an almost contact manifold if there exists a $(1-1)$ tensor field $\varphi$, a unit vector field $\xi$ (called the Reeb vector field) and a 1-form $\eta$ such that

$$
\varphi^{2} X=-X+\eta(X) \xi
$$

From which it is easy to verify $\varphi \xi=0, \eta \circ \varphi=0$. A Riemannian metric $g$ on $M$ is said to be an associated metric if it satisfies

$$
g(\varphi X, \varphi Y)=g(X, Y)-\eta(X) \eta(Y)
$$


for any vector fields $X Y$ on $M$. A Riemannian manifold $M^{2 n+1}$ together with an almost contact metric structure $(\varphi, \xi, \eta, g)$ is said to be an almost contact metric manifold. We remark that an almost contact metric structure on a Riemannian manifold $M^{2 n+1}$ may be regarded as a reduction of the structure group $M$ to $U(n)$. For such a manifold, we can always define a 2form $\varphi$ by $\varphi(X, Y)=g(X, \varphi Y)$ which is known as the fundamental 2-form. The almost contact metric structure is said to be normal if $[\varphi, \varphi](X, Y)+2 d \eta(X, Y) \xi=0$, for any vector field $X, Y$ on $M$, where

$$
[\varphi, \varphi](X, Y)=[\varphi X, \varphi Y]+\varphi^{2}[X, Y]-\varphi[\varphi X, Y]-\varphi[X, \varphi Y]
$$

An almost contact metric manifold is said to be an almost Kenmotsu manifold if it satisfies $d \eta=0$ and $d \varphi=2 \eta \wedge \varphi$. Further, a normal almost Kenmotsu manifold is said to be a Kenmotsu manifold, and this normality condition is expressed as

$$
\left(\nabla_{X} \varphi\right) Y=g(\varphi X, Y) \xi-\eta(Y) \varphi X
$$

for any vector field $X, Y$ on $M$. The following formulas also hold for a Kenmotsu manifold [13]

$$
\begin{gathered}
\nabla_{X} \xi=X-\eta(X) \xi \\
R(X, Y) \xi=\eta(X) Y-\eta(Y) X \\
Q \xi=-2 n \xi
\end{gathered}
$$

where $R$ is the curvature tensor.

Next, we recall the notion of a $\beta$-Kenmotsu manifold which is a slight extension of the Kenmotsu manifold. An almost contact metric manifold $M(\varphi, \xi, \eta, g)$ is said to be $\beta$-Kenmotsu if it satisfies

$$
\left(\nabla_{X} \varphi\right) Y=\beta\{g(\varphi X, Y) \xi-\eta(Y) \varphi X\}
$$

for some smooth function $\beta$ on $M$. Kenmotsu manifolds appear as particular case of $\beta$-Kenmotsu manifolds with $\beta=1$. Regarding the existence of such manifold we recall the following (e.g, see [1]).

Lemma 1. The warped product $M=\mathbb{R} \times{ }_{\sigma} N$, is a $\beta$-Kenmotsu manifold with $\beta=\sigma^{\prime} / \sigma$, where $\mathbb{R}$ is the real line and $N$ is a Kähler manifold.

Recall that a vector field $V$ on a contact manifold is said to be a contact vector field if

$$
£_{V} \eta=f \eta
$$

for some smooth function $f$ on $M$. The contact vector field $V$ is called strict when $f=0$. Finally, we recall some formulas involving Lie-derivatives along an arbitrary vector field $V$. First of all, using the well-known commutation formula (see p. 23 of [19]):

$$
\left(£_{V} \nabla_{X} g-\nabla_{X} £_{V} g-\nabla_{[V, X]} g\right)(Y, Z)=-g\left(\left(£_{V} \nabla\right)(X, Y), Z\right)-g\left(\left(£_{V} \nabla\right)(X, Z), Y\right),
$$

we deduce

$$
\left.\left(\nabla_{X} £_{V} g\right)(Y, Z)\right)=g\left(\left(£_{V} \nabla\right)(X, Y), Z\right)+g\left(\left(£_{V} \nabla\right)(X, Z), Y\right) .
$$

The following formulas are also known (see [19, p.23]):

$$
\begin{gathered}
\left(£_{V} R\right)(X, Y) Z=\left(\nabla_{X} £_{V} \nabla\right)(Y, Z)-\left(\nabla_{Y} £_{V} \nabla\right)(X, Z), \\
£_{V} \nabla_{X} Y-\nabla_{X} £_{V} Y-\nabla_{[V, X]} Y=\left(£_{V} \nabla\right)(X, Y) .
\end{gathered}
$$




\section{KENMOTSU MANIFOLD SATISFYING $£_{\xi} Q=0$}

Recently, Cho-Kimura [6] proved that if the Reeb vector field of a 3-dimensional Kenmotsu manifold leaves the Ricci operator invariant, then it is locally isometric to $\mathbb{H}^{3}(-1)$. Extending this in higher diemnsions we prove

Theorem 1. Let $M(\varphi, \xi, \eta, g)$ be a Kenmotsu manifold of dimension $(2 n+1)$. If the Reeb vector field $\xi$ of $M$ leaves the Ricci operator $Q$ invariant, then $M$ is Einstein.

The proof of the theorem follows from the following lemma.

Lemma 2. For any Kenmotsu manifold of dimension $(2 n+1)$ the following are valid

(i) $\left(£_{\xi} Q\right) Y=-2 Q Y-4 n Y$,

(ii) $\left(\nabla_{\xi} Q\right) Y=-2 Q Y-4 n Y$.

Proof. By virtue of (3), we have

$$
\left(£_{\xi} g\right)(Y, Z)=g\left(\nabla_{Y} \xi, Z\right)+g\left(\nabla_{Z} \xi, Y\right)=2\{g(Y, Z)-\eta(Y) \eta(Z)\}
$$

Taking covariant differentiation of (9) along an arbitrary vector field $X$ and the use of (3) gives

$$
\left(\nabla_{X} £_{\xi} g\right)(Y, Z)=2\{2 \eta(X) \eta(Y) \eta(Z)-g(X, Y) \eta(Z)-g(X, Z) \eta(Y)\}
$$

Making use of this in (6), we obtain

$$
\begin{aligned}
g\left(\left(£_{\xi} \nabla\right)(X, Y), Z\right)+g\left(\left(£_{\xi} \nabla\right)(X, Z), Y\right) & =2\{2 \eta(X) \eta(Y) \eta(Z) \\
& -g(X, Y) \eta(Z)-g(X, Z) \eta(Y)\}
\end{aligned}
$$

By a combinatorial combination, we deduce

$$
\begin{aligned}
g\left(\left(£_{\xi} \nabla\right)(Y, Z), X\right)+g\left(\left(£_{\xi} \nabla\right)(Y, X), Z\right) & =2\{2 \eta(Y) \eta(Z) \eta(X) \\
& -g(Y, Z) \eta(X)-g(Y, X) \eta(Z)\}, \\
g\left(\left(£_{\xi} \nabla\right)(Z, X), Y\right)+g\left(\left(£_{\xi} \nabla\right)(Z, Y), X\right) & =2\{2 \eta(Z) \eta(X) \eta(Y) \\
& -g(Z, X) \eta(Y)-g(Z, Y) \eta(X)\} .
\end{aligned}
$$

Subtracting the first equation from the addition of the last two equations provides

$$
\left(\left(£_{\xi} \nabla\right)(Y, Z)=2\{\eta(Y) \eta(Z)-g(Y, Z) \xi\}\right.
$$

Differentiating (10) covariantly along $X$ and using (3), we find

$$
\begin{aligned}
\left(\nabla_{X} £_{\xi} \nabla\right)(Y, Z)=2\{g(X, Y) \eta(Z) \xi & +g(X, Z) \eta(Y) \xi+g(Y, Z) \eta(X) \xi \\
& -g(Y, Z) X+\eta(Y) \eta(Z) X-3 \eta(X) \eta(Y) \eta(Z) \xi\} .
\end{aligned}
$$


Utilizing this in (7), we obtain

$$
\left(£_{\xi} R\right)(X, Y) Z=2\{g(X, Z) Y-g(Y, Z) X+\eta(Y) \eta(Z) X-\eta(X) \eta(Z) Y\}
$$

Contracting (11) over $X$ with respect to an orthonormal frame of the tangent space of $M$, we deduce

$$
\left(£_{\xi} S\right)(Y, Z)=4 n\{\eta(Y) \eta(Z)-g(Y, Z)\} .
$$

Next, we take the Lie derivative of $S(Y, Z)=g(Q Y, Z)$, to get

$$
\left(£_{\xi} S\right)(Y, Z)=\left(£_{\xi} g\right)(Q Y, Z)+g\left(\left(£_{\xi} Q\right) Y, Z\right) .
$$

On the other hand, replacing $Y$ by $Q Y$ in (9) and using (5), we obtain

$$
\left(£_{\xi} g\right)(Q Y, Z)=2\{g(Q Y, Z)+2 n \eta(Y) \eta(Z)\}
$$

Using (14) and (13) in (12) we obtain $\left(£_{\xi} Q\right) Y=-2 Q Y-4 n Y$. This completes the proof of (i). Taking into account of (3) we observe that

$$
\begin{aligned}
\left(£_{\xi} Q\right) Y & =£_{\xi} Q Y-Q £_{\xi} Y=\nabla_{\xi} Q Y-\nabla_{Q Y \xi}-Q \nabla_{\xi} Y+Q \nabla_{Y} \xi \\
& =\left(\nabla_{\xi} Q\right) Y-Q Y-2 n \eta(Y) \xi+Q Y+2 n \eta(Y) \xi=\left(\nabla_{\xi} Q\right) Y .
\end{aligned}
$$

Using this in (i) we complete the proof of (ii).

For a 3-dimensional Riemannian manifold it is known that the Ricci curvature determines the curvature completely and the curvature tensor can be explicitely expressed as

$$
\begin{aligned}
R(X, Y) Z=S(Y, Z) X & -S(X, Z) Y+g(Y, Z) Q X-g(X, Z) Q Y \\
& -\frac{r}{2}\{g(Y, Z) X-g(X, Z) Y\} .
\end{aligned}
$$

Now, if a 3-dimensional Kenmotsu manifold $M$ satisfies $£_{\xi} Q=0$, then from (i) of the above Lemma $Q X=-2 X$. Using this in (15) we see that $M^{3}$ is of constant curvature -1 . Thus, we have the following (see also [6]).

Corollary 1. A 3-dimensional Kenmotsu manifold satisfies $£_{\xi} Q=0$ if and only if $M^{3}$ is locally isometric to a Hyperbolic space $\mathbb{H}^{3}(-1)$.

\section{KenMOTSU METRIC AS A RiCCI SOLITON}

In [9] the author proved that if a Kenmotsu 3-metric represents a Ricci soliton, then it is of constant curvature -1 . Here we extend this result in higher dimensions and prove the following assertion.

Theorem 2. Let $M(\varphi, \xi, \eta, g)$ be a Kenmotsu manifold of dimension $(2 n+1)(>3)$. If $g$ represents a Ricci soliton, then the soliton is expanding. Moreover, the soliton is trivial (Einstein) if either (i) $V$ is a contact vector field, or (ii) the Reeb vector field $\xi$ leaves the scalar curvature invariant. 
Proof. First, differentiating (5) along an arbitrary vector field $X$ and recalling (3) we deduce

$$
\left(\nabla_{X} Q\right) \xi=-Q X-2 n X .
$$

Differentiating (1), using it in (6) and by a straightforward combinatorial computation we obtain

$$
g\left(\left(£_{V} \nabla\right)(X, Y), Z\right)=\left(\nabla_{Z} S\right)(X, Y)-\left(\nabla_{X} S\right)(Y, Z)-\left(\nabla_{Y} S\right)(X, Z) .
$$

Taking $\xi$ instead of $Y$ in the preceding equation and then recalling (16) and (ii) of Lemma 3.1 provides

$$
\left(£_{V} \nabla\right)(Y, \xi)=2 Q Y+4 n Y .
$$

Covariant differentiation of (17) along $X$ and the use of (3), (17) leads to

$$
\left(\nabla_{X} £_{V} \nabla\right)(Y, \xi)+\left(£_{V} \nabla\right)(Y, X)-2 \eta(X)(Q Y+2 n Y)=2\left(\nabla_{X} Q\right) Y .
$$

Making use of this in (7) and since $£_{V} \nabla$ is a symmetric operator, one can deduce

$$
\begin{aligned}
\left(£_{V} R\right)(X, Y) \xi=2\left\{\eta(X) Q Y-\eta(Y) Q X+\left(\nabla_{X} Q\right) Y\right. & \left.-\left(\nabla_{Y} Q\right) X\right\} \\
& +4 n\{\eta(X) Y-\eta(Y) X\} .
\end{aligned}
$$

We now set $Y=\xi$ in the foregoing equation and making use of (16) and (ii) of Lemma 3.1 to achieve $\left(£_{V} R\right)(X, \xi) \xi=0$. On the other hand, Lie differentiating the formula (follows from (4)): $R(X, \xi) \xi=-X+\eta(X) \xi$ along $V$ provides

$$
\left(£_{V} R\right)(X, \xi) \xi+R\left(X, £_{V} \xi\right) \xi+R(X, \xi) £_{V} \xi=\left\{\left(£_{V} \eta\right) X\right\} \xi+\eta(X) £_{V} \xi .
$$

Moreover, from (4) it follows that $R(X, \xi) Y=g(X, Y) \xi-\eta(Y) X$. Using this, (4) and $\left(£_{V} R\right)(X, \xi) \xi=0$ in $(18)$, we ultimately obtain

$$
g\left(X, £_{V} \xi\right) \xi-2 \eta\left(£_{V} \xi\right) X=\left\{\left(£_{V} \eta\right) X\right\} \xi
$$

Now from the soliton equation (1) along with (5) shows that

$$
\left(£_{V} \eta\right) X-g\left(X, £_{V} \xi\right)-2(\lambda+2 n) \eta(X)=0 .
$$

Also, Lie differentiating $g(\xi, \xi)=1$ along $V$ and taking into account (1), (5) provides

$$
\eta\left(£_{V} \xi\right)=\lambda+2 n
$$

Using (20) and (21) in (19) it follows that $\lambda=-2 n$. Hence the soliton is expanding. Next, we assume that $V$ is a contact vector field. Then by using $£_{V} \eta=f \eta, \lambda=-2 n$ and (20) it follows that $£_{V} \xi=f \xi$. Scalar product of this with $\xi$ and using (21) implies that $f=\lambda+2 n=0$. Thus, $£_{V} \xi=0$, and hence $V$ is strict. Replacing $Y$ by $\xi$ in (8) and using (3), $£_{V} \xi=0=£_{V} \eta$, we have

$$
\begin{aligned}
\left(£_{V} \nabla\right)(X, \xi) & =£_{V} \nabla_{X} \xi-\nabla_{[V, X]} \xi=£_{V}(X-\eta(X) \xi)-£_{V} X+\eta\left(£_{V} X\right) \xi \\
& =£_{V} X-\eta(X) £_{V} \xi-\left\{\left(£_{V} \eta\right) X\right\} \xi-\eta\left(£_{V} X\right) \xi-£_{V} X+\eta\left(£_{V} X\right) \xi=0 .
\end{aligned}
$$

Finally, using (17), we can conclude that $M$ is Einstein. This completes the proof of (i). Next, we assume that the the Reeb vector field $\xi$ leaves the scalar curvature $r$ invariant. This means that $£_{\xi} r=\xi r=0$. On the other hand, tracing (i) of Lemma 2, we have

$$
\xi r=-2\{r+2 n(2 n+1)\} .
$$


From which it follows that $r=-2 n(2 n+1)$. Hence, the scalar curvature $r$ is constant. Next, we recall the following integrability formula ([5], [17])

$$
£_{V} r=-\Delta r+2 \lambda r+2|Q|^{2},
$$

for a Ricci soliton, where $\Delta r=-\operatorname{div} D r$. Since $r$ is constant (22) shows that $|Q|^{2}=-\lambda r=-2 n r$. By virtue of this and $r=-2 n(2 n+1)$, we compute

$$
\begin{aligned}
|Q+2 n I|^{2} & =|Q|^{2}+2 n r+2 n r+4 n^{2}(2 n+1) \\
& =2 n r+4 n^{2}(2 n+1)=2 n\{r+2 n(2 n+1)\}=0 .
\end{aligned}
$$

Since the length of the symmetric tensor $Q+2 n I$ vanishes, we must have $Q=-2 n I$. This shows that $M$ is Einstein and we complete the proof.

We have mentioned earlier that the warped product $\mathbb{R} \times{ }_{\sigma} N^{2 n}$, where $N^{2 n}$ is a Kähler manifold of dimension $2 n$ and $\sigma(t)=c e^{2 t}$ is the warping function, naturally admits Kenmotsu structure. From this, we have the following result.

Corollary 2. If the metric of the warped product $\mathbb{R} \times_{\sigma} N^{2 n}$ represents a Ricci soliton then it is necessarily expanding.

Remark 1. The above corollary shows that there exists examples of non-compact expanding Ricci solitons. In dimension 3, this has been derived explicitely in [9].

\section{RicCi ALMOST SOLITON AND KENMOTSU METRIC}

In this section, we study Kenmotsu metric as a Ricci almost soliton. First, we construct a Kenmotsu metric that admits a Ricci almost soliton. The existence of non-compact Ricci almost soliton has been established by Pigola et. al [16] on some certain class of warped product manifolds. Following Lemma 1.1 of [16] we can construct the following example of a Ricci almost soliton.

Example 1. Consider the warped product $\mathbb{R} \times_{\sigma(t)} \mathbb{H}^{n}$ with metric $g=d t^{2}+\sigma^{2}(t) g_{0}$, where $g_{0}$ is the standard metric on the hyperbolic space $\mathbb{H}^{n}$. Let $\sigma(t)=\cosh t$, then the warped product $\mathbb{R} \times_{\sigma(t)} \mathbb{H}^{n}$ becomes Einstein manifold with Ricci tensor $S^{M}=-n g$ and it admits a Ricci almost soliton $(g, \nabla f, \lambda)$ with $f(x, t)=\sinh t$ and $\lambda(x, t)=\sinh t-n$.

From this example we have the following one.

Example 2. Let $M^{2 n+1}=\mathbb{R} \times \cosh t \mathbb{C H}^{2 n}$ with metric $g=d t^{2}+\left(\cosh ^{2} t\right) g_{0}$, where $g_{0}$ is the standard metric on the complex hyperbolic space $\mathbb{C H}^{2 n}$. Then $M^{2 n+1}$ becomes Einstein manifold with Ricci tensor $S^{M}=-2 n g$ (follows from Lemma 1.1 of [16]). Consequently, $\left(M^{2 n+1}, g, \nabla f, \lambda\right)$ is a Ricci almost soliton with $f(x, t)=\sinh t$ and $\lambda(x, t)=\sinh t-2 n$.

Example 3. Let $N^{2 n}$ be a complete Einstein Kaehler manifold with $S^{N}=-(2 n-1) g_{0}$. We consider the warped product $\left(M^{2 n+1}, g\right)=\left(\mathbb{R} \times \cosh t \mathbb{N}^{2 n}, d t^{2}+(\cosh t)^{2} g_{0}\right)$. Then it follows from Lemma 1.1 of [16] that $\left(M^{2 n+1}, g\right)$ is Einstein with $S^{M}=-2 n g$. Since $M$ is complete, by the result of Kanai, there exists a function $f$ on $M$ without critical points satisfying $\nabla^{2} f=$ $-f g$ (see Theorem $D$ of [12]). Now if we choose $\lambda=-2 n-f$, then it is easy to see that $\left(M^{2 n+1}, g, \nabla f, \lambda\right)$ is a nontrivial gradient Ricci almost soliton. 
From, the last two examples it is evident that the metrics are not Kenmotsu. In fact, by Lemma 1 it follows that the warped product $\mathbb{R} \times \cosh t \mathbb{C H}^{2 n}$ is a $\beta$-Kenmotsu manifold with $\beta=\tanh t$. So, we consider a $D$-conformal deformation [14] on the warped product to transform the warped product metric into a Kenmotsu metric. Let

$$
\bar{g}=\sigma g+(1-\sigma) \eta \otimes \eta
$$

where $\sigma$ is positive function depending only on the direction of $\xi$. By virtue of Lemma 2.2 of ( [2]) it is easy to see that the resulting manifold $\bar{M}(\varphi, \xi, \eta, \bar{g})$ is $\bar{\beta}$-Kenmotsu, where $\bar{\beta}=\beta+\frac{\xi \sigma}{2 \sigma}$. Next, we choose $\bar{\beta}$ in such a way that the manifold $\bar{M}$ under consideration will be a Kenmotsu. So, we look for $\sigma$ satisfying:

$$
\beta+\frac{\xi \sigma}{2 \sigma}=1
$$

Making use of a local parametrization that is $\xi=\frac{\partial}{\partial t}$, the foregoing equation can be written as

$$
\frac{\partial}{\partial t}(\ln \sigma)=2(1-\beta)
$$

Solving this we get $\sigma=e^{2 t} /(\cosh t)^{2}$. Finally, using this and (25), equation (26) takes the form

$$
\bar{g}=e^{2 t} g+d t^{2} .
$$

This is the desired Kenmotsu metric.

The above example motivates us to consider Kenmotsu metric as a gradient Ricci almost soliton. Thus, the equation (2) holds for a smooth function $\lambda$.

Theorem 3. If the metric of a Kenmotsu manifold $M(\varphi, \xi, \eta, g)$ of dimension $(2 n+1)$ represents a gradient Ricci almost soliton, then it is $\eta$-Einstein and the soliton is expanding. Moreover, if $M$ is complete and the Reeb vector field $\xi$ leaves the scalar curvature $r$ invariant, then $M$ is locally isometric to a hyperbolic space $\mathbb{H}^{2 n+1}$, and the potential (soliton) function, upto an additive constant, can be expressed as a linear combination of cosh $t$ and sinh $t$.

Proof. By virtue of (2) and the well known expression of the curvature tensor $R(X, Y)=$ $\left.\left[\nabla_{X}, \nabla_{Y}\right]-\nabla_{[} X, Y\right]$, we deduce

$$
R(X, Y) D f=\left(\nabla_{Y} Q\right) X-\left(\nabla_{X} Q\right) Y+(X \lambda) Y-(Y \lambda) X
$$

Taking $\xi$ instead of $Y$ in the foregoing equation, recalling (ii) of Lemma 3.1 and (16) provides

$$
\begin{aligned}
R(X, \xi) D f & =\left(\nabla_{\xi} Q\right) X-\left(\nabla_{X} Q\right) \xi+(X \lambda) \xi-(\xi \lambda) X \\
& =-2 Q X-4 n X+Q X+2 n X+(X \lambda) \xi-(\xi \lambda) X \\
& =-Q X-2 n X+(X \lambda) \xi-(\xi \lambda) X .
\end{aligned}
$$

Making use of $R(X, \xi) Y=g(X, Y) \xi-\eta(Y) X$ (follows from (4)) the foregoing equation implies that

$$
g(X, D f-D \lambda) \xi-\{(\xi f)-(\xi \lambda)\} X=-Q X-2 n X
$$


Inner product of of this equation with $\xi$ and using (5) shows

$$
D f-D \lambda=\{(\xi f)-(\xi \lambda\} \xi .
$$

Thus, using (26) in (25) provides

$$
\{(\xi f)-(\xi \lambda\} \eta(X) \xi-\{(\xi f)-(\xi \lambda)\} X=-Q X-2 n X .
$$

Next, we contract equation (24) over $X$ to deduce

$$
Q D f=\frac{1}{2} D r-2 n D \lambda \text {. }
$$

By virtue of this and (5) one can obtain $\frac{1}{4 n}(\xi r)=(\xi \lambda)-(\xi f)$. But from (ii) of Lemma 3.1, we have $(\xi r)=-2(r+2 n(2 n+1))$. Therefore, $(\xi f)-(\xi \lambda)=\frac{r}{2 n}+2 n+1$. Using this (27) can be expressed as

$$
S(X, Y)=\left(\frac{r}{2 n}+1\right) g(X, Y)-\left(\frac{r}{2 n}+2 n+1\right) \eta(X) \xi .
$$

This shows that $M$ is $\eta$-Einstein. Now, if $\xi$ leaves the scalar curvature $r$ invariant, then $\xi r=0$. Consequently, $r=-2 n(2 n+1)$. By virtue of this, (28) shows that $Q X=-2 n X$, i.e., $M$ is Einstein. Since $r=-2 n(2 n+1)$, we have $(\xi \lambda)=(\xi f)$ and therefore $D f=D \lambda$. Hence, equation (2) may be exhibited as

$$
\nabla_{X} D \lambda=(2 n+\lambda) X
$$

Applying Tashiro's theorem [18] we conclude that $M$ is isometric to the hyperbolic space $\mathbb{H}^{2 n+1}$. Since $\nabla_{\xi} \xi=0$ (follows from (3)), we deduce from (29) that $\xi(\xi \lambda)=2 n+\lambda$. But we know [13] that a Kenmotsu manifold $M$ of dimension $2 n+1$ is locally isometric to the warped product $(-\varepsilon, \varepsilon) \times_{c e^{t}} N$, where $N$ is a Kähler manifold of dimension $2 n$ and $(-\varepsilon, \varepsilon)$ is an open interval. Using the local parametrization: $\xi=\frac{\partial}{\partial t}$ (where $t$ denotes the coordinate on $(-\varepsilon, \varepsilon))$ we obtain

$$
\frac{d^{2} \lambda}{d t}=2 n+\lambda
$$

Its solution can be exhibited as $\lambda=A \cosh t+B \sinh t-2 n$, where $A, B$ are constants on $M$. This completes the proof.

Example 4. Let $\left(N, J, g_{0}\right)$ be a Kähler manifold of dimension $2 n$. Consider the warped product $(M, g)=\left(\mathbb{R} \times{ }_{\sigma} N, d t^{2}+\sigma^{2} g_{0}\right)$, where $t$ is the coordinate on $\mathbb{R}$. We set $\eta=d t, \xi=\frac{\partial}{\partial t}$ and the tensor field $\varphi$ is defined on $\mathbb{R} \times_{\sigma} N$ by $\varphi X=J X$ for vector field $X$ on $N$ and $\varphi X=0$ if $X$ is tangent to $\mathbb{R}$. Then it is easy to testify (see [13]) that the warped product $\mathbb{R} \times{ }_{\sigma} N, \sigma^{2}=c e^{2 t}$, with the structure $(\varphi, \xi, \eta, g)$ is an almost Kenmotsu manifold, by Lemma 1. Thus, if we take $\sigma(t)=c e^{t}$, then $M$ becomes a Kenmotsu manifold. Further, we set $N=C H^{2 n}$, then $N$ being Einstein, the Ricci tensor of $M$ such that $S=-2 n g$. Define $f(t)=k e^{t}, k>0$. Then it is easy to verify that $(M, \nabla f, g)$ is a Ricci almost soliton with $\lambda=k e^{t}-2 n$. Similarly, we may also construct many examples of Ricci almost solitons by taking different potential functions on the warped product.

Next, we consider a special type of Ricci almost soliton on Kenmotsu manifolds in which the potential vector field $V$ is a pointwise collinear with the Reeb vector filed $\xi$. This type of problem has been considered by the author within the framework of contact metric manifolds in [10]. 
Theorem 4. Let $M(\varphi, \xi, \eta, g)$ be a Kenmotsu manifold of diemnsion $(2 n+1)$. If $g$ represents a non-trivial Ricci almost soliton such that the potential vector field $V$ is pointwise collinear with the Reeb vector field, then it is $\eta$-Einstein.

Proof. By hypothesis $V=k \xi$, for some smooth function $k$ on $M$. Differentiating this along an arbitrary vector field $X$ and using (3), we have

$$
\nabla_{X} V=(X k) \xi+k(X-\eta(X) \xi)
$$

In view of (30) the soliton equation transforms to

$$
2 S(X, Y)+2(k-\lambda) g(X, Y)+(X k) \eta(Y)+(Y k) \eta(X)-2 k \eta(X) \eta(Y)=0 .
$$

Setting $X=Y=\xi$ in (31) and using (5), we have $\xi k=2 n+\lambda$. Further, taking $\xi$ instead of $Y$, using (5) and $\xi k=2 n+\lambda$ shows that $X k=(2 n+\lambda) \eta(X)$. Making use of this in (31), we have

$$
Q X=(\lambda-k) X-(2 n+\lambda-k) \eta(X) \xi .
$$

Tracing the foregoing equation provides

$$
\frac{r}{2 n}=(\lambda-k)-1
$$

By virtue of (33), (32) can be written as

$$
Q X=\left(\frac{r}{2 n}+1\right) X-\left(\frac{r}{2 n}+2 n+1\right) \eta(X) \xi .
$$

This shows that $M^{2 n+1}$ is $\eta$-Einstein and we complete the proof.

Remark 2. If $k$ is constant, then from $X k=(\lambda+2 n) \eta(X)$, we see that $\lambda=-2 n$ and which is constant. Thus from (33) follows that $\xi r=0$. Then from (ii) of Lemma 3.1, we have $(\xi r)=$ $-2(r+2 n(2 n+1))$. Hence $r=-2 n(2 n+1)$. Making use of this in (33) we see that $k=0$, and in this case the soliton becomes trivial.

\section{REFERENCES}

[1] Alegre P., Blair D.E., Carriazo A. Generalized Sasakian-space-forms. Israel J. Math. 2004, 141, $157-183$.

[2] Alegre P., Carriazo A. Generalized Sasakian space forms and conformal changes of the metric. Results Math. 2011, 59 (3-4), 485-493. doi:10.1007/s00025-011-0115-z

[3] Barros A., Ribeiro Jr E. Some characterizations for compact almost Ricci solitons. Proc. Amer. Math. Soc. 2012,140 (3), 213-223. doi:10.1090/S0002-9939-2011-11029-3

[4] Barros A., Batista R., Ribeiro Jr E. Compact almost Ricci solitons with constant scalar curvature are gradient. Monatsh. Math. 2014, 174 (1), 29-39. doi:10.1007/s00605-013-0581-3

[5] Chow B., Knopf D. The Ricci flow: An introduction. Mathematical Surveys and Monographs. 110, American Mathematical Society, 2004.

[6] Cho J.T., Kimura M. Reeb flow symmetry on almost contact three-manifolds. Differential Geom. Appl. 2014, 35, 266-276.

[7] Derdzinski A. Ricci solitons. Preprint 2017 - arXiv:1712.06055v1. 
[8] Friedan D. Nonlinear models in $2+\varepsilon$ dimensions. Ann. Physics 1985, 163, 318-419.

[9] Ghosh A. Kenmotsu 3-metric as a Ricci soliton. Chaos Solitons Fractals 2011, 44, 647-650.

[10] Ghosh A. An $\eta$-Einstein Kenmotsu metric as a Ricci soliton. Publ. Math. Debrecen 2013, 82 (3-4), 591-598.

[11] Hamilton R.S. The Ricci flow on surfaces. Mathematics and general relativity. Contemp. Math., Amer. Math. Soc. 1988, 71, 237-262.

[12] Kanai M. On a differential equation characterizing a Riemannian manifold. Tokyo J. Math. 1983, 6(1), $143-151$.

[13] Kenmotsu K. A class of almost contact Riemannian manifolds. Tohoku Math. J. 1972, 24, 93-103.

[14] Marrero J.C. The local structure of trans-Sasakian manifolds. Ann. Mat. Pura Appl. 1992, 162, 77-86.

[15] Perelman G. The entropy formula for the Ricci flow and its geometric applications. Preprint 2002. arXiv: math/0211159v1.

[16] Pigola S., Rigoli M., Rimoldi M., Setti A. Ricci almost solitons. Ann. Sc. Norm. Super. Pisa Cl. Sci. 2011, 10 (5), 757-799.

[17] Sharma R. Certain results on K-contact and $(k, \mu)$-contact manifolds. J. Geom. 2008, 89, 138-147.

[18] Tashiro Y. Complete Riemannian manifolds and some vector fields. Trans. Amer. Math. Soc. 1965, 117, $251-275$.

[19] Yano K. Integral formulas in Riemannian Geometry, Marcel Dekker, New York, 1970.

Received 22.05.2018

Гош А. Солітон Річиі і майже солітон Річиі в рамках многовиду Кенмочу // Карпатські матем. публ. — 2019. — Т.11, №1. — С. 59-69.

Ми доводимо, спочатку, що якшо векторне поле Ріба $\xi$ многовида Кенмоцу $М$ залишає оператор $Q$ інваріантним, то $M \in$ Айнштайнівським. Аалі ми вивчаємо многовид Кенмоцу, метрика якого зображує солітон Річчі, і доводимо, що він $є$ поширюючим. Більше того, солітон є тривіальним (Айнштайнівським), якщо або (i) $V \in$ контактним векторним полем або (ii) векторне поле Ріба $\xi$ залишає скалярну кривизну інваріантною. Нарешті, доведено, шо якщо метрика многовиду Кенмоцу зображає деякий градієнтний майже солітон Річчі, то цей многовид $€ \eta$-Айнштайнівським і цей солітон $є$ поширюючим. Ми також демонструємо деякі приклади многовиду Кенмоцу, які допускають майже солітони Річчі.

Ключові слова і фрази: многовид Кенмоцу, майже солітон Річчі, викривлений добуток. 